\title{
Noise-enhanced heterodyning in bistable systems
}

\author{
M.I. Dykman, ${ }^{*}$ G.P. Golubev ${ }^{\dagger}$ D.G. Luchinsky, ${ }^{\dagger}$ P.V.E. McClintock, N.D. Stein, \\ and N.G. Stocks ${ }^{\ddagger}$ \\ School of Physics and Materials, Lancaster University, Lancaster, LA1 4YB, United Kingdom
}

(Received 27 July 1993)

\begin{abstract}
A new form of heterodyning is reported in which a heterodyne signal at the difference frequency between an input signal and a reference signal can be enhanced by adding noise. The underlying mechanism is closely related to that of stochastic resonance. The dependences of the heterodyne signal and the signal-to-noise ratio on the amplitudes and frequencies of the input and reference signals have been investigated. Noise-induced enhancement of the heterodyning has been demonstrated both for white noise and for high-frequency noise (with the power spectrum centered at the frequency of the reference signal) added at the input.
\end{abstract}

PACS number(s): 05.40.+j, 02.50.-r, 84.30.Qi, 84.30.Vn

\section{INTRODUCTION}

One of the important physical problems of information processing and transfer is how to control the signalto-noise ratio (SNR). Usually this ratio decreases with increasing intensity of noise. However, under certain circumstances it behaves in the opposite way. The phenomenon of the noise-induced increase (and subsequent decrease) of the signal and of the SNR in the system was called stochastic resonance [1]. It has attracted much attention recently (see [2]). Most of the data on stochastic resonance have been obtained for bistable systems driven by noise and by a low-frequency periodic force. The onset of stochastic resonance in these systems is related to the fact that the probabilities $W_{n m}$ of transitions between coexisting stable states $(n, m=1,2)$ increase exponentially, in the case of Gaussian noise, with increasing noise intensity $D: W_{n m} \propto \exp \left(-G_{n} / D\right)$ for large $G_{n} / D$, where $G_{n}$ is the characteristic activation energy of the transition from the state $n$ (in the case of thermally activated escape from a potential well $G_{n}$ is the depth of the well, $G_{n} \equiv \Delta U_{n}$, and $D$ is temperature). A low-frequency external periodic force $A \cos \Omega t$ modulates the activation energies, $G_{n} \rightarrow G_{n}+G_{n}^{\prime} A \cos \Omega t$, and as a result the transition probabilities are modulated too, with the strength of the modulation being determined by the ratio $A / D$, so that this modulation can be comparatively strong even for small amplitudes of the force. In turn, the modulation of $W_{n m}$ gives rise to a modulation of the populations of the stable states. For a particle in a double-well potential with equal well depths the mechanism of the latter modulation is obvious: the force periodically makes one

\footnotetext{
* Permanent address: Department of Physics, Stanford University, Stanford, CA 94305.

${ }^{\dagger}$ Permanent address: VNIIMS, Andreevskaya nab. 2, Moscow, 117965, Russia.

${ }^{\ddagger}$ Present address: Department of Engineering, University of Warwick, Coventry, CV4 7AL, UK.
}

of the wells deeper than the other, and the system occupies it with a larger probability. Periodic modulation of the populations of the wells results in a comparatively strong modulation of the average value of the coordinate of the system $x$, the amplitude of the oscillations being proportional to the difference $x_{1}-x_{2}$ in the values of the coordinate in the stable states $x_{n}$.

The above mechanism comes into play through fluctuational transitions, i.e., through the noise, and the amplitude of the periodic signal correspondingly increases with increasing noise intensity. Because it does so exponentially fast in a certain range of $D$, the SNR may be expected to increase with $D$ too. The mechanism is most effective, provided (i) the stationary populations of the states in the absence of the driving are nearly equal to each other [3], and (ii) the frequency of the force is small: it has to be very much smaller than the reciprocal relaxation time of the system $t_{r}^{-1}$ in particular, so that the transitions have a chance to occur over the period $2 \pi / \Omega\left(W_{n m} \ll t_{r}^{-1}\right.$ for small noise intensities $)$.

The frequency-selective response of bistable systems, and also the fact that the SNR increases with increasing noise intensity, makes it interesting to apply the idea of stochastic resonance to heterodyning so as to obtain a form of the phenomenon that is enhanced rather than suppressed by noise. In heterodyning, two highfrequency fields, one of them being an input signal and the other a reference signal, are mixed nonlinearly to generate a heterodyne signal at the difference frequency. Mixing of this kind occurs quite generally in any nonlinear system. In most cases, however, the addition of noise will result in a decrease in the amplitude of the heterodyne signal (and its SNR) because the frequency response of the system becomes correspondingly broadened. Nonetheless, we show below that, in bistable systems of the kind that exhibit stochastic resonance (SR) [1-3], the heterodyne signal (and SNR) can sometimes be enhanced by an increase in the noise intensity. (Note that, although bistability is not necessary for SR, which can also occur in monostable systems [4], it is for the bistable case that the largest noise-induced increases in 
the signal and SNR have been observed.) In this paper we outline the theory and report the observation of noise-enhanced heterodyning (NEH) in bistable systems by means of analog electronic simulation.

The theory of NEH is developed in Sec. II. In Sec. III the analog electronic simulations undertaken to test the theory are described, together with relevant experimental details. The results are presented, compared with the theoretical predictions, and discussed in Sec. IV. Conclusions are drawn in Sec. V.

\section{THEORY}

We shall illustrate the onset of NEH by consideration of a simple model of an overdamped bistable system driven by three time-dependent forces that stand, respectively, for the reference and input signals, and the noise. The motion of the system is described by the equation

$$
\begin{aligned}
\frac{d x}{d t}= & -U^{\prime}(x)+A_{\mathrm{ref}} x \cos \omega_{0} t \\
& +A_{\mathrm{in}}(t) \cos \left[\omega_{0} t+\phi(t)\right]+f(t) .
\end{aligned}
$$

Here, the term proportional to $A_{\text {ref }}$ is the high-frequency reference signal corresponding to a local oscillator of frequency $\omega_{0}$ (the corresponding force is applied multiplicatively), and that proportional to $A_{\text {in }}(t)$ is the modulated high-frequency input signal (applied additively). The functions $A_{\text {in }}(t)$ and $\phi(t)$ are slowly varying as compared with $\cos \omega_{0} t$, and it is their variation in time that has to be revealed via heterodyning. The heterodyning can be characterized by the low-frequency signal at the output, $x^{(\mathrm{sl})}(t)=\overline{x(t)}$ (the overbar stands for averaging over the period $\left.2 \pi / \omega_{0}\right)$, for $A_{\text {in }}=$ const and $\phi=\Omega t+$ const, with $\Omega \ll \omega_{0}$, i.e., for a monochromatic input signal whose frequency $\omega_{0}+\Omega$ is slightly different from the frequency $\omega_{0}$.

We shall assume that the double-well potential of the system $U(x)$ has equally deep wells, which is most appropriate for standard stochastic resonance, and we choose it to be of the form

$$
U(x)=-\frac{1}{2} x^{2}+\frac{1}{4} x^{4} .
$$

The minima of the potential (2) (the stable states of the system) lie at $x_{n}=(-1)^{n}, n=1,2$, and the characteristic (dimensionless) relaxation time of the system $t_{r} \equiv 1 / U^{\prime \prime}\left(x_{n}\right)=1 / 2$. The analysis of heterodyning in bistable systems is not limited to the particular form of Eqs. (1) and (2). The effect occurs in both overdamped and underdamped systems. However, the explicit expressions take on a simpler form for the model (1), (2), and they are further simplified in the case where the frequencies of the input and reference signals are high compared with the reciprocal relaxation time of the system,

$$
\omega_{0} \gg t_{r}^{-1} \quad\left(t_{r}=\frac{1}{2}\right) .
$$

The term $f(t)$ in (1) is a random force. It is supposed to be a zero-mean Gaussian noise. We will allow for a form of this noise that has two independent components, at low and high frequencies, respectively, with the latter being randomly modulated vibrations at frequency $\omega_{0}$ :

$$
f(t)=f_{\mathrm{LF}}(t)+f_{\mathrm{HF}}(t), f_{\mathrm{HF}}(t)=\operatorname{Re}\left[\tilde{f}_{\mathrm{HF}}(t) \exp \left(-i \omega_{0} t\right)\right]
$$

The power spectrum $\Phi_{\mathrm{LF}}(\omega)$ of the low-frequency noise $f_{\mathrm{LF}}(t)$ is assumed to be flat up to $\omega \sim \omega_{c} \gg t_{r}^{-1}\left(\omega_{c}\right.$ may be small compared to $\left.\omega_{0}\right)$. The power spectrum $\Phi_{\mathrm{HF}}(\omega)$ of the high-frequency noise $f_{\mathrm{HF}}(t)$ is assumed to be centered at $\omega_{0}$ and also flat over a range greatly exceeding $t_{r}^{-1}$ (such a noise being of interest because it often occurs in practice, e.g., resulting from the scattering of a radio signal):

$$
\begin{aligned}
& \Phi_{\mathrm{LF}}(\omega)=D_{\mathrm{LF}} / \pi \text { for } \omega \lesssim \omega_{c} \\
& \Phi_{\mathrm{HF}}(\omega)=D_{\mathrm{HF}} / \pi \text { for }\left|\omega^{2}-\omega_{0}^{2}\right| \lesssim 2 \omega_{0} \omega_{c} \quad\left(\omega_{c} \gg t_{r}^{-1}\right) \\
& \Phi(\omega)=\lim _{\tau \rightarrow \infty}(4 \pi \tau)^{-1}\left|\int_{-\tau}^{\tau} d t f(t) \exp (i \omega t)\right|^{2}
\end{aligned}
$$

The power spectra $\Phi(\omega)$ decay smoothly outside the indicated regions.

For $\omega_{0} \gg t_{r}^{-1}$ the motion of the system consists of fast oscillations at frequency $\omega_{0}$ (and its overtones) superimposed on a slow motion. Correspondingly, in the spirit of [5], we shall seek the solution to (1) in the form

$$
\begin{aligned}
x= & x^{(0)}+x^{(1)}, \\
x^{(1)}= & \omega_{0}^{-1}\left\{A_{\mathrm{ref}} x^{(0)} \sin \omega_{0} t+A_{\mathrm{in}}(t) \sin \left[\omega_{0} t+\phi(t)\right]\right. \\
& \left.-\operatorname{Im}\left[\tilde{f}_{\mathrm{HF}}(t) \exp \left(-i \omega_{0} t\right)\right]\right\}
\end{aligned}
$$

where the last term refers to the case of a high-frequency noise. On substituting (6) into (1) we obtain an equation for $x^{(0)}$. This equation contains terms oscillating at frequencies $\omega_{0}, 2 \omega_{0}$, and $3 \omega_{0}$, and also slowly varying terms. We shall assume the amplitudes of the forces $A_{\text {ref }}, A_{\text {in }}(t)$ and also the noise intensity $D$ sufficiently small that the amplitudes of the vibrations of $x$ at the overtones of $\omega_{0}$ can be treated as small perturbations. Then the main contribution to $x^{(0)}$ comes from the slowly varying terms. The equation for the slow part of the coordinate takes on the form

$$
\begin{aligned}
& x^{(0)} \approx x^{(\mathrm{sl})}, \\
& \dot{x}^{(\mathrm{sl})}=-U^{\prime}\left(x^{(\mathrm{sl})}\right)+A(t) \sin \phi(t)+f^{(0)}(t), \\
& A(t)=\frac{A_{\mathrm{ref}}}{2 \omega_{0}} A_{\mathrm{in}}(t),
\end{aligned}
$$

where

$$
\begin{aligned}
& f^{(0)}(t)=f_{\mathrm{LF}}(t)-\frac{A_{\mathrm{ref}}}{2 \omega_{0}} \operatorname{Im} \tilde{f}_{\mathrm{HF}}(t), \\
& \Phi^{(0)}(\omega) \approx D / \pi \text { for } \omega \lesssim \omega_{c}, \\
& D=D_{\mathrm{LF}}+\left(A_{\mathrm{ref}}^{2} / 2 \omega_{0}^{2}\right) D_{\mathrm{HF}} .
\end{aligned}
$$

Here, $\Phi^{(0)}(\omega)$ is the power spectrum of the force $f^{(0)}(t)$; 
we have assumed that $\operatorname{Re} \tilde{f}_{\mathrm{HF}}(t)$ and $\operatorname{Im} \tilde{f}_{\mathrm{HF}}(t)$ are statistically independent of each other and have the same intensity, and also that they are statistically independent of $f_{\mathrm{LF}}(t)$. In deriving $(7)$ we have neglected corrections $\sim\left(\omega_{0} t_{r}\right)^{-1}$ due to the nonlinearity of the potential $U(x)$ : the dominant contribution to the right-hand side of (7) comes from the dependence on $x$ of the force corresponding to the reference signal; also the spectral density $\Phi_{\mathrm{HF}}(\omega)$ in the range of low frequencies $\omega \lesssim t_{r}^{-1}$ has been supposed very small and ignored.

For $A(t), \phi(t)$ varying slowly over the time $t_{r}$ the dynamics of the system described by Eqs. (2), (7), and (8) has already been investigated in detail in the context of stochastic resonance. It depends strongly on the interrelation between the effective noise intensity $D$ and the depths of the potential wells for slow motion $\Delta U_{n}=U_{\max }-U\left(x_{n}\right)\left(U_{\max }\right.$ is the value of the potential in the local maximum). For the potential (2) $\Delta U_{1}=\Delta U_{2} \equiv \Delta U=1 / 4$. For very small $D / \Delta U$ the motion of the system is exclusively intrawell, and the amplitude of the vibrations induced by the input signal depends weakly on the intensity of the noise, so that the SNR decreases with increasing $D$. For higher $D / \Delta U$ the interwell transitions come into play, and the SNR may increase with increasing $D$.

In the particular case of a periodic input signal, $A_{\text {in }}=$ const, $\phi=\Omega t+$ const, the average smooth part of the coordinate $\left\langle x^{(\mathrm{sl})}(t)\right\rangle$ varies periodically at the low modulation frequency $\Omega$, and the power spectrum of the coordinate contains $\delta$ spikes at the frequencies $n \Omega$ on top of the broad spectrum. For small $A \propto A_{\text {in }}$ the vibrations of $\left\langle x^{(\mathbf{s l})}(t)\right\rangle$ are practically monochromatic, $\left\langle x^{(\mathrm{sl})}(t)\right\rangle=a_{0}+a \sin \psi(t)$, with their amplitude $a$ and phase $\psi$ given by the expression

$a \sin \psi(t)=-A \operatorname{Im}\left[\chi(\Omega) e^{-i \phi(t)}\right], \phi(t)=\Omega t+$ const.

For small noise intensities $(\Delta U \gg D)$ the susceptibility $\chi(\Omega)$ in the case of a symmetric double-well potential $U(x)$ is of the form $[3,6]$

$$
\chi(\Omega)=\frac{1}{t_{r}^{-1}-i \Omega}+\frac{W}{4 D} \frac{\left(x_{2}-x_{1}\right)^{2}}{W-i \Omega}
$$

Here, $W \equiv W_{12}+W_{21} \propto \exp (-\Delta U / D)$ is the relaxation rate of the populations of the stable states. It is seen from (9), (10) that, since $W$ increases sharply with the noise intensity $D$, the amplitude of the heterodyne signal at the difference frequency $\Omega$ increases with noise intensity in a certain range of $D$, too.

This noise-induced enhancement of the heterodyning can be characterized by the dependence on $D$ of the ratio $R$ of the intensity (area) of the $\delta$ spike in the power spectrum of the system at frequency $\Omega$, induced by the force proportional to $A$, to the value of the power spectrum at the same frequency for $A=0$. For $\Omega t_{r} \ll 1$ but for arbitrary $\Omega / W$ the expression for the signal-to-noise ratio $R$ is of the form (cf. [6])

$$
\begin{aligned}
& R=\pi \frac{A^{2}\left(x_{2}-x_{1}\right)^{2}}{16 D^{2}} \frac{W^{2}+\Omega^{2} t_{r}^{2} \tilde{D}^{2}}{W+\Omega^{2} t_{r}^{2} \tilde{D}}, \\
& \tilde{D}=4 D\left(x_{2}-x_{1}\right)^{-2}, \\
& \Omega, \tilde{D} \ll t_{r}^{-1}, W \ll \tilde{D}
\end{aligned}
$$

to lowest order in the small parameters in (11). It follows from (11) (and also from the more general expression for the SNR) that, in the range of noise intensities where

$$
\Omega \tilde{D} t_{r} \lesssim W \lesssim t_{r}^{-1}
$$

the SNR increases with increasing noise intensity. The increase is quite sharp, being nearly exponential. This means that noise-enhanced heterodyning would be expected to arise in a bistable system, whether driven by a low- or a high-frequency noise (or both).

An intuitive picture of the physical mechanism underlying NEH is as follows. The reference force provides a modulation of the potential $(-1 / 2) A_{\mathrm{ref}} x^{2} \cos \omega_{0} t$ which is even in the coordinate, and therefore does not break the symmetry of the system. The phases of the vibrations $\delta x_{\text {ref }}(t)$ about the minima of the wells caused by this signal differ by $\pi$ (i.e., the vibrations are in counterphase). The situation is quite different for the input signal $A_{\text {in }}$. This signal, being additive, breaks the symmetry of the potential, and the amplitude and phase of the periodic vibrations of the coordinate $\delta x_{\text {in }}(t)$ at the signal frequency $\omega_{\text {in }}$ are the same in the absence of the reference signal for both wells (the vibrations are in phase). When both forces are present, they combine nonlinearly, giving rise to potential terms oscillating at combination frequencies, and in particular to a term at the difference frequency $\left|\omega_{0}-\omega_{\text {in }}\right|$ which is proportional, approximately, to the product of $\delta x_{\text {ref }} \times \delta x_{\mathrm{in}}$. This term defines the slow motion of the system. Obviously, it is antisymmetric: its sign is opposite for the two wells [of course, we have to allow for cross terms in the time-dependent part of the potential $-(1 / 2) x^{2} A_{\text {ref }} \cos \omega_{0} t-x A_{\text {in }} \cos \left(\omega_{\text {in }} t+\psi_{\text {in }}\right)$, where $\psi_{\text {in }}$ is a constant; the corresponding slow terms are also antisymmetric].

The amplitude of the fast oscillations induced in the system by the superimposed reference and input signals varies slowly in time, as the effects of the individual signals tend alternately to reinforce or cancel each other. These amplitude variations occur in antiphase between the two wells: when the amplitude of the fast oscillation is relatively large in one well, it is relatively small in the other, and vice versa. The particular well for which the fast vibrations are of the larger amplitude at a given time will also be the one from which fluctuational transitions are most likely to occur. This is because the center of oscillations is then shifted furthest towards the saddle (much like the case of quasimonochromatic noise in the adiabatic limit: see [7]). In the presence of noise, therefore, there is an enhanced probability of an interwell transition occurring once per half cycle of the difference frequency, thereby effectively amplifying the heterodyne signal in very much the same way as a low-frequency additive signal can be amplified in conventional bistable SR [2]. 


\section{ANALOG ELECTRONIC EXPERIMENTS ON NOISE-ENHANCED HETERODYNING}

The occurrence of NEH has been investigated experimentally by means of a conventionally designed [8] ana$\log$ electronic circuit model of the system (1), (2). The technique is in essence extremely simple: an electronic model of the stochastic differential equations under study is built, using operational amplifiers, multipliers, and other standard analog components to perform the required mathematical operations of addition, subtraction, multiplication, division, integration, etc. Its response to stochastic and periodic forces is analyzed with the aid of a digital data processor.

Figure 1(a) shows a block diagram of the circuit used to model an overdamped bistable Duffing oscillator driven by external noise $f(t)$ in the presence of a signal $A_{\text {in }}^{\prime} \cos \left(\omega_{0}^{\prime}+\Omega^{\prime}\right) t^{\prime}$ and a multiplicatively applied reference signal $A_{\text {ref }}^{\prime} \cos \omega_{0}^{\prime} t^{\prime}$. Here $A_{\text {in }}^{\prime}, A_{\text {ref }}^{\prime}$ are the amplitudes of the signals in volts, $f^{\prime}\left(t^{\prime}\right)$ is the actual value of the noise applied to the circuit, and $\omega_{0}^{\prime}$ and $t^{\prime}$ are the real frequency and time.

The actual differential equation for the voltage $x^{\prime}$ in the circuit was of the form

$$
\begin{aligned}
R_{1} C_{1} \frac{d x^{\prime}}{d t^{\prime}}= & \frac{R_{9}}{R_{6}} \frac{R_{1}}{R_{3}} x^{\prime}-\frac{R_{9}}{100 R_{7}} \frac{R_{5} R_{1}}{R_{4} R_{3}} x^{3} \\
& +\frac{R_{9}}{10 R_{8}} \frac{R_{1}}{R_{3}} A_{\mathrm{ref}}^{\prime} x^{\prime} \cos \omega_{0}^{\prime} t^{\prime} \\
& +\frac{R_{1}}{R_{2}} A_{\mathrm{in}}^{\prime} \cos \left(\omega_{0}^{\prime}+\Omega^{\prime}\right) t^{\prime}+f^{\prime}\left(t^{\prime}\right),
\end{aligned}
$$

where we have chosen

$$
\begin{aligned}
& R_{1}=R_{2}=R_{3}=R_{4}=R_{7}=R_{8}=10 \mathrm{k} \Omega, \\
& R_{5}=R_{9}=50 \mathrm{k} \Omega, \quad R_{6}=200 \mathrm{k} \Omega, \quad C_{1}=30 \mathrm{nF}
\end{aligned}
$$

(cf. [8] for details of the operation of analog electronic circuits used to simulate fluctuating nonlinear systems; note that the output of the AD534 [9] analog multipliers is internally divided by 10 ). To investigate experimentally the enhancement of heterodyning by white noise this circuit was driven by noise from a feedback shift-register noise generator and by two sinusoidal periodic forces from Hewlett-Packard Model 3325B frequency synthesizers.

The high-frequency noise needed for a second series of experiments was produced by filtering broadband exponentially correlated noise $\eta\left(t^{\prime}\right)$ from a feedback shiftregister noise generator with a circuit simulating an underdamped harmonic oscillator frequency $\omega_{0}^{\prime}$, shown in Fig. 1(b). The output of the filter was quasimonochromatic noise (QMN) [7]; to suppress its low-frequency components, which were undesirable in the present context, the QMN was passed through the additional highpass filter formed by $R_{D} C_{D}$. The corresponding differential equations for the noise at the output of the filters are of the form

$$
R_{10} C_{2} R_{13} C_{3} \frac{d^{2} f^{\prime}}{d t^{\prime 2}}+\frac{R_{10}}{R_{12}} R_{13} C_{3} \frac{d f^{\prime}}{d t^{\prime}}+\frac{R_{10}}{R_{11}} \frac{R_{15}}{R_{14}} f^{\prime}=s\left(t^{\prime}\right)
$$

$R_{D} C_{D} \frac{d}{d t^{\prime}}\left[\eta\left(t^{\prime}\right)-s\left(t^{\prime}\right)\right]=s\left(t^{\prime}\right)$

\section{(a)}

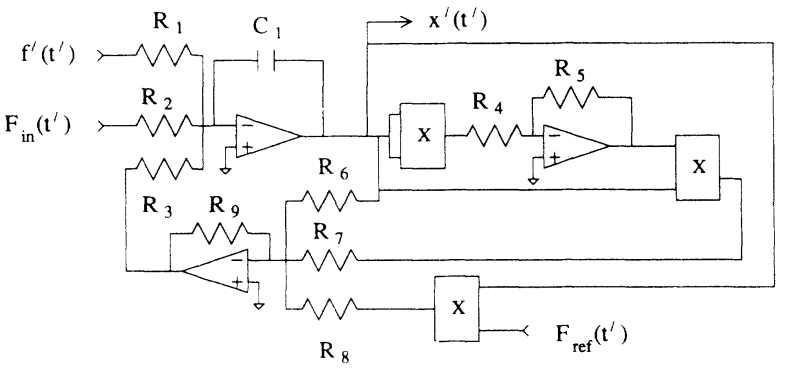

(b)

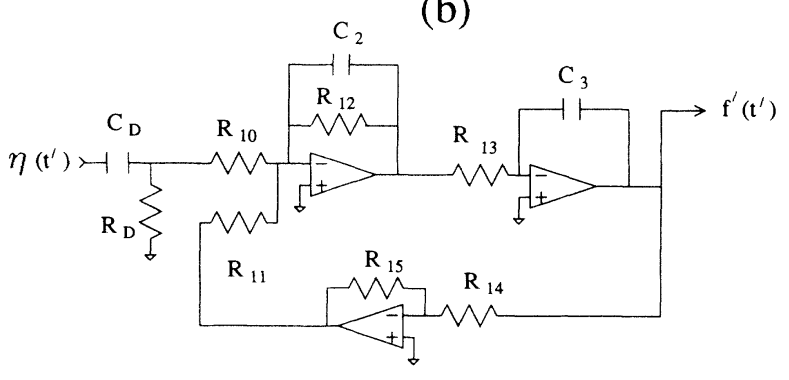

(c)

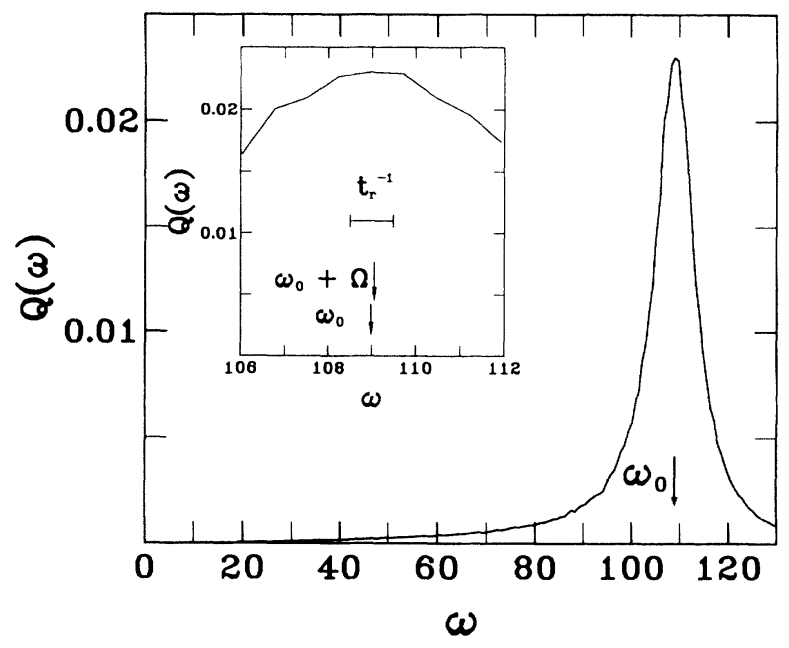

FIG. 1. Block diagrams of the electronic circuits used in the simulations. (a) Circuit modeling an overdamped particle (1) in the potential (2): $F_{\text {ref }}\left(t^{\prime}\right)=A_{\text {ref }}^{\prime} \cos \omega_{0}^{\prime} t^{\prime}$ and $F_{\text {in }}\left(t^{\prime}\right)=A_{\text {in }}^{\prime} \cos \left(\omega_{0}^{\prime}+\Omega^{\prime}\right) t^{\prime}$; the noise input $f^{\prime}\left(t^{\prime}\right)$ can either be exponentially correlated quasiwhite noise or the output of circuit (b), which is high-frequency narrow-band noise. (b) Circuit used to convert quasiwhite noise into band-limited noise. (c) Spectral density of the band-limited noise measured at the output of the circuit (b). The inset shows the same spectrum expanded near its maximum: the bar shows the smallness of the reciprocal relaxation time compared to the width of the peak; and the arrows indicate the abscissa positions of $\omega_{0}$ and $\left(\omega_{0}+\Omega\right)$. 
with

$$
\begin{aligned}
& R_{10}=R_{11}=R_{13}=R_{14}=R_{15}=22 \mathrm{k} \Omega, \\
& R_{12}=220 \mathrm{k} \Omega, \quad C_{2}=C_{3}=0.5 \mathrm{nF}, \\
& R_{D}=100 \mathrm{k} \Omega, \quad C_{D}=1 \mathrm{nF}, \quad \tau_{D}=R_{D} C_{D} .
\end{aligned}
$$

The correlation time $\tau_{N}$ of the noise $\eta\left(t^{\prime}\right)$ was much smaller than all other integration times in the circuits

$$
\begin{aligned}
& \tau_{N} \leq \tau_{2} \ll \tau_{2} / \Gamma^{\prime} \ll 4 \tau_{1}, \\
& \tau_{1}=R_{1} C_{1}, \quad \tau_{2}=R_{10} C_{2}=R_{13} C_{3}, 2 \Gamma^{\prime}=R_{13} / R_{12} .
\end{aligned}
$$

When written in terms of $\tau_{1}, \tau_{2}, \Gamma^{\prime}$, with account taken of the interrelations between the resistances $R_{i}$, the equations for $x^{\prime}, f^{\prime}, \eta$ take the form

$$
\begin{aligned}
& 4 \tau_{1} \frac{d x^{\prime}}{d t^{\prime}}=x^{\prime}-x^{3}+2 A_{\mathrm{ref}}^{\prime} x^{\prime} \cos \omega_{0}^{\prime} t^{\prime} \\
& +4 A_{\text {in }}^{\prime} \cos \left(\omega_{0}^{\prime}+\Omega^{\prime}\right) t^{\prime}+4 f^{\prime}\left(t^{\prime}\right), \\
& \tau_{2}^{2} \frac{d^{2} f^{\prime}}{d t^{\prime 2}}+2 \Gamma^{\prime} \tau_{2} \frac{d f^{\prime}}{d t^{\prime}}+f^{\prime}=s\left(t^{\prime}\right), \\
& \tau_{D} \frac{d}{d t^{\prime}}\left[\eta\left(t^{\prime}\right)-s\left(t^{\prime}\right)\right]=s\left(t^{\prime}\right) .
\end{aligned}
$$

After the scaling

$$
\begin{aligned}
& t^{\prime} \rightarrow 4 \tau_{1} t, \quad \omega_{0}^{\prime} \rightarrow \omega_{0} / 4 \tau_{1}, \Omega^{\prime} \rightarrow \Omega / 4 \tau_{1}, \\
& A_{\mathrm{ref}}^{\prime} \rightarrow A_{\mathrm{ref}} / 2, \quad A_{\mathrm{in}}^{\prime} \rightarrow A_{\mathrm{in}} / 4, \\
& f^{\prime}\left(t^{\prime}\right) \rightarrow f_{\mathrm{HF}}(t) / 4, \quad \Gamma^{\prime} \rightarrow\left(\tau_{2} / 4 \tau_{1}\right) \Gamma, \\
& \left(\tau_{1} / \tau_{2}\right) \rightarrow \tilde{\omega}_{0} / 4, \quad\left(\tau_{1} / \tau_{D}\right) \rightarrow \omega_{D} / 4,
\end{aligned}
$$

and changing $x^{\prime} \rightarrow x$, Eq. (16) goes over into (1) with the effective intensity of the high-frequency noise $f_{\mathrm{HF}}(t)$ at the frequency $\omega_{0}$ being given to an excellent approximation by

$$
D_{\mathrm{HF}}=\frac{\tilde{\omega}_{0}^{2} D_{\eta}\left(\tilde{\omega}_{0} / \omega_{D}\right)^{2}}{4 \Gamma^{2}\left[1+\left(\tilde{\omega}_{0} / \omega_{D}\right)^{2}\right]}
$$

where $D_{\eta}$ is the effective intensity of the noise $\eta\left(t^{\prime}\right)$ on the input of the circuit Fig. 1(b). In the experiment $\omega_{0}$ was nearly equal to $\tilde{\omega_{0}}$. The actual form of the input bandlimited noise is shown in Fig. 1(c). It is evident from this figure and the above analysis that the power spectrum of high-frequency noise has the form of that for a damped oscillator centered at $\omega_{0}$ with a suppressed low-frequency wing. In addition to the band-limited noise the circuit was again driven by two sinusoidal periodic forces from Hewlett-Packard 3325B frequency synthesizers.

In each case, the response of the circuit, a time-varying voltage representing $x(t)$, was digitized (12-bit precision) typically in 1024-word (1k) or $2 \mathrm{k}$ blocks and analyzed using a Nicolet LAB80 data-processor computer. The required spectral densities were computed by means of a standard fast-Fourier-transform (FFT) routine. The signal-to-noise ratio, defined in the usual way [10] as the ratio of the peak height at the signal frequency to the broadband noise level at the same frequency, was then extracted by measuring the contents of relevant processor "bins."

\section{RESULTS AND DISCUSSION}

\section{A. Heterodyning enhanced by white noise}

In Fig. 2 the theory and experimental data for the dependence of the amplitude of the heterodyne signal $S=a^{2}(9)$ and the SNR $R(11)$ on noise intensity are compared for the case of the exponentially correlated noise. In fact, the cutoff frequency $\omega_{c}$ of the noise was high, and it exceeded $\omega_{0}$ in some of the experiments; no dependence on $\omega_{c}$ was observed for $\omega_{c} \gg t_{r}^{-1}$, so it would be reasonable to call this noise white [we notice that the effect of its high-frequency components is minor: according to (8) its contribution is of the order of $\left(A_{\text {ref }} / \omega_{0}\right)^{2} \sim 5 \times 10^{-4}$ in the experiment]. It is clearly seen that the dependence of the signal amplitude on $D$ is of the form of an asymmetric resonant curve, which is extremely steep on the small- $D$ side. This form is typical for stochastic resonance [2]. For small $D$, where the system is effectively confined to one well of the potential, the signal magnitude corresponds in effect to the value it would have for heterodyning in a single-well nonlinear potential, and is extremely small. The dependence of the SNR on $D$ (inset) is also similar to that seen in stochastic resonance: for very small $D$ where the interwell transitions do not come into play, $R$ decreases with increasing $D$, but then it displays a sharp increase. We notice that the maximum of $R$ vs $D$ is shifted to higher noise intensities compared to that of the amplitude of the heterodyne signal. The experimental data are in good qualitative and quantitative agreement with the simple theory outlined in Sec. II (curves), without any adjustable parameters [we have used expressions that allow for corrections $\sim D / \Delta U$ omitted in (10), (11), cf. [6]].

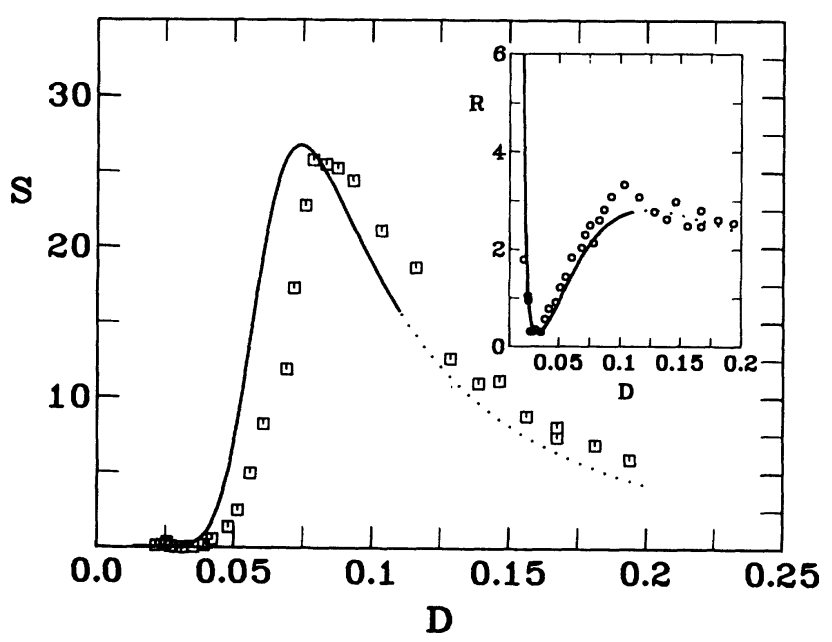

FIG. 2. Normalized heterodyne signal $S$ and signal-tonoise ratio $R$ (inset) as functions of the noise intensity $D$ for $\omega_{0}=1.885, \Omega=0.008$. The value of $S$ is determined by the ratio of the squared amplitude of the heterodyne signal to the squared amplitude of the scaled input signal $A(t)$ [see Eq. (7)]. The boxes are the experimental data; the curves represent the theory, which has been dotted in the range of large $D$ where it is only qualitative. 


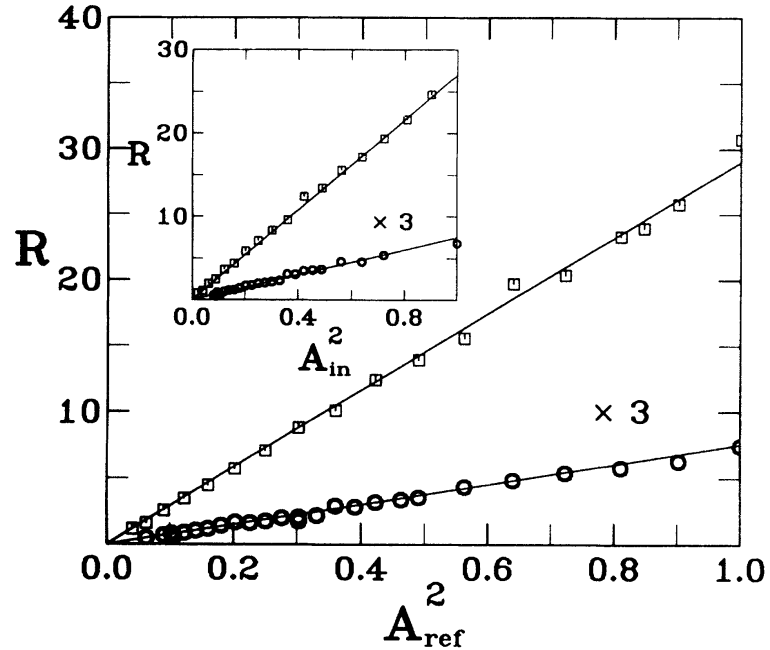

FIG. 3. Experimental dependence of the SNR, $R$, on the squared amplitude of the reference signal for the noise intensities $D=0.015$ (boxes) and $D=0.14$ (circles); the carrier frequency $\omega_{0}=1.885$, and the difference frequency $\Omega=0.008$. The inset shows the dependence of $R$ on the squared amplitude of the input signal, under the same conditions.

It follows from (7), (11) that the SNR, for a given effective noise intensity $D$, should be proportional to the squared amplitudes of both the reference and input signals, and inversely proportional to the squared reference frequency $\omega_{0}$. These predictions have been observed to hold over a broad range of parameters. The Fig. 3 inset demonstrates the linear dependence of the experimental values of the SNR at the difference frequency on the squared amplitude of the high-frequency input signal $A_{\text {in }}^{2}$, a feature that is very important from the viewpoint of

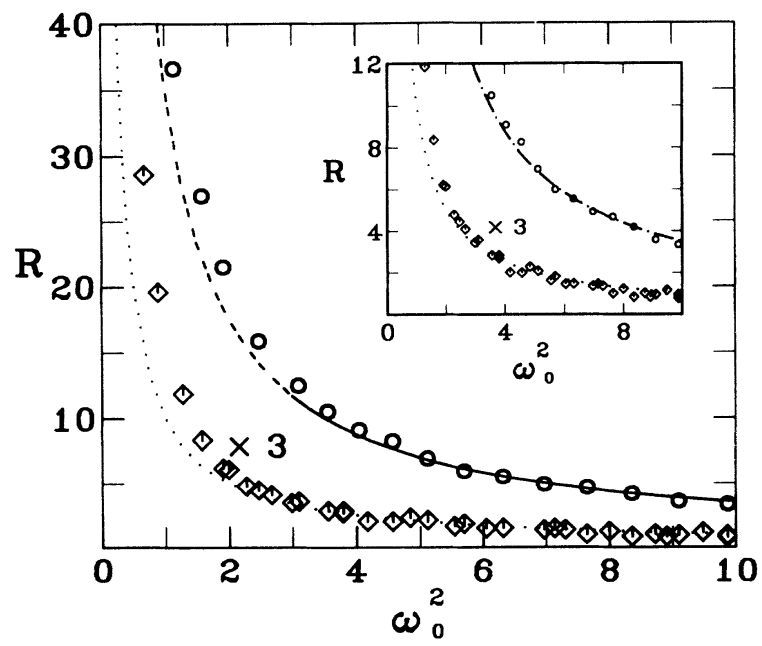

FIG. 4. The dependence of SNR on the squared frequency $\omega_{0}$ of the reference signal for the noise intensities $D=0.015$ (circles) and $D=0.14$ (lozenges); the difference frequency $\Omega=0.008$. The dashed and solid lines are proportional to $\omega_{0}^{-2}$ [the theory refers to the range $\omega_{0} \gg 1$, for the potential (2)]. The inset shows the same data with a magnified ordinate scale. signal processing. The lower set of data refers to a value of the noise intensity $D$ corresponding approximately to the maximum of $R$ vs $D$, whereas the upper one corresponds to $D$ lying on the initial descending part of the $R(D)$ curve. Quite similarly (Fig. 3, main section), the proportionality of $R$ to the squared amplitude of the reference signal $A_{\text {ref }}^{2}$ has been clearly observed throughout broad ranges of the noise intensity $D$ and of the frequencies $\omega_{0}, \Omega$ (we notice that for very small difference frequencies $\Omega$ there arises a nonlinearity of $R$ vs $A_{\text {ref }}^{2}, A_{\text {in }}^{2}$ for small $D$, similar to the situation in stochastic resonance [3]). The dependence of the SNR on $\omega_{0}$ is seen from Fig. 4 to be very close to $\omega_{0}^{-2}$ up to $\omega_{0} t_{r} \approx 1$ for the system under consideration.

\section{B. Heterodyning enhanced by a high-frequency noise}

The experimental results presented in Figs. 2-4 relate to the case of a broadband (white) noise at the input of the circuit. In heterodyning it is often important to allow for the effects of a noise whose spectral density peaks around the frequency $\omega_{0}$ of the signal. For this reason, the enhancement of heterodyning by high-frequency noise is of the utmost interest in view of possible applications. It follows from the theoretical results discussed above that this case has much in common with the white-noise one, provided that the power spectrum of the noise is flat over a range exceeding $t_{r}^{-1}$ and decays steeply at small frequencies $\sim t_{r}^{-1}$, the only difference being in the dependence (8) of the effective noise intensity $D$ on the squared amplitude of the reference signal.

It is clear from the inset of Fig. 1(c) that the power spectrum of the high-frequency noise in the experiment was indeed flat over a range greatly exceeding $t_{r}^{-1}$, and that $t_{r}^{-1}$ greatly exceeded the frequency detuning between the reference and the carrier frequencies. Near the maximum the shape of this power spectrum could be described by a Lorentzian, of half-width $\Gamma$. After demodulation this spectrum converts into exponentially correlated noise with correlation time $\sim \Gamma^{-1}$. Given that

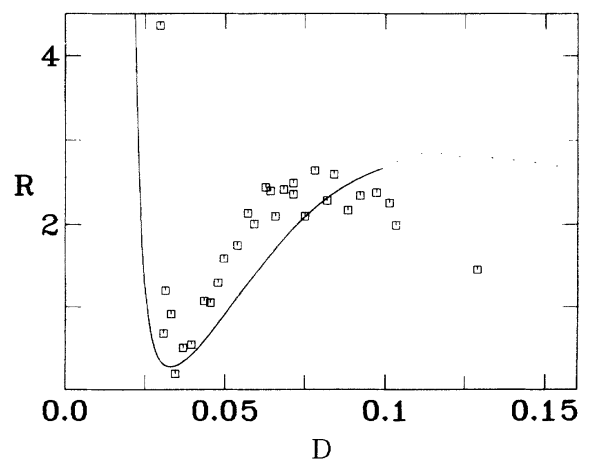

FIG. 5. Signal-to-noise ratio $R$ as a function of noise intensity $D$, demonstrating noise-enhanced heterodyning for high-frequency noise. The boxes and solid line represent experiment and theory for $\omega_{0}=109.091, \Omega=0.0017$. Beyond its range of validity, where the theory is merely qualitative, it is shown dotted. 
$\Gamma \gg t_{r}^{-1} \gg \Omega$, this noise may therefore be considered as white for our circuit. In these limits we can apply the theory of the noise-enhanced heterodyning described in Sec. II. The data on the SNR in heterodyning for highfrequency noise are shown in Fig. 5. They are similar to the data for the white-noise-driven system: the SNR for the heterodyne signal clearly displays noise enhancement, and the data are in qualitative agreement with the theory. The discrepancy with the theory is attributable to the presence of relatively weak low-frequency noise at the output of the circuit, Fig. 1(b).

\section{The frequency dependence of NEH}

Of special interest is the dependence of the SNR on the modulation frequency $\Omega$. It follows from (11) that in the interesting range where $R$ increases sharply with noise intensity, i.e., in the range where $W>\Omega t_{r} \tilde{D}$ and $D \lesssim \Delta U, R$ also increases quite sharply with $\Omega$, from the value that corresponds to the SNR in the neglect of the intrawell motion, $R=R_{0} \approx \pi A^{2} W / 4 D \tilde{D}$ for very small $\Omega$, up to the value that corresponds to the SNR in the neglect of the interwell transitions, $R \approx \pi A^{2} / 4 D$. A simple way to suppress such frequency dispersion in the SNR is to apply two-state filtering, passing the output through a Schmitt trigger, so that the continuous system is effectively replaced by a two-state one, i.e., the coordinate is supposed to take on either of two values that correspond to the positions of the stable states, and it takes on one or the other of them when the real continuous coordinate is in the vicinity of the corresponding stable state (such filtering is often considered in the context of stochastic resonance, cf. $[1,2])$. For $\Omega \ll t_{r}^{-1}$ the theoretical value of the SNR at the output of a two-state filter is given approximately by the value of $R$ (11) for $\Omega=0$ (or for $t_{r}=0$ : the intrawell relaxation is "infinitely fast"). The experimental frequency dependence of $R$ on $\Omega$ for the filtered signal (boxes) and without filtering (circles) is shown in Fig. 6. It can be seen that, for small $\Omega / \Omega_{r}$, the dispersion is almost eliminated by the filtration; the remaining dispersion (of opposite sign) seen at higher frequencies is attributable to a frequency dependence of the modulation amplitude of the activation energies of the transitions.

\section{CONCLUSION}

We have shown that, in close analogy to conventional SR [2], NEH in a bistable system can produce a very substantial enhancement of the heterodyne signal over

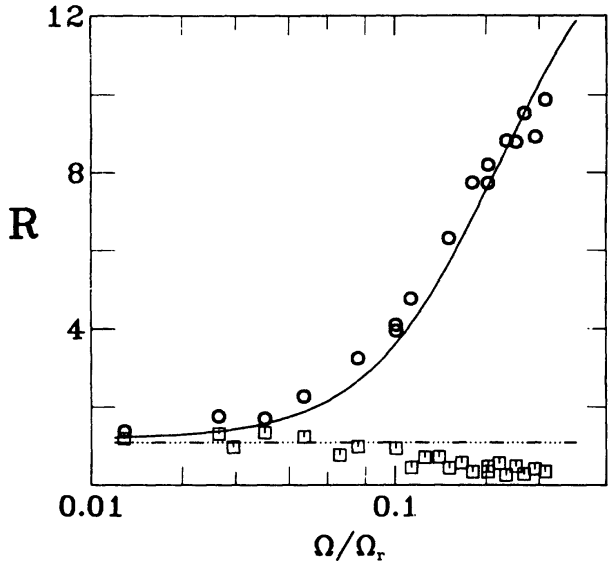

FIG. 6. The frequency dispersion of the SNR for noiseenhanced heterodyning before (circles) and after (boxes) applying two-state filtering; noise intensity $D=0.055$, the carrier frequency $\omega_{0}=5.02$. The solid and dashed-dotted lines represent theory.

that obtained for heterodyning in, e.g., a single-well nonlinear system. The same is not true, however, of the SNR. Notwithstanding the huge noise-induced rises seen in $R(D)$ in NEH, and in conventional SR, the SNR does not quite reach the value it would have for the same $D$ in a single-well system. Nonetheless, we note that NEH offers a method by which the SNR can be protected against changes in the noise on the signal, if the average operating point is chosen (see Fig. 2 or 5 ) to be very close to but slightly beyond the maximum in $R(D)$. In applications where it is important for the SNR to be stable, this could prove to be a useful feature.

In conclusion, we have demonstrated, theoretically and experimentally, that bistable systems can be used to obtain heterodyning in which not only the amplitude of the heterodyne signal at the output, but also the signal-tonoise ratio, increase dramatically with increasing intensity of the noise at the input.

\section{ACKNOWLEDGMENTS}

We are grateful to $\mathrm{N}$. Abraham for a valuable discussion. The work was supported by the Science and Engineering Research Council (U.K.), by the European Community, by the Royal Society of London, and by the Gosstandart of Russia. M.I.D., G.P.G., and D.G.L. gratefully acknowledge extremely warm hospitality at Lancaster University.
[1] R. Benzi, A. Sutera, and A. Vulpiani, J. Phys. A 14, L453 (1981); C. Nicolis, Tellus 34, 1 (1982); R. Benzi, G. Parisi, A. Sutera, and A. Vulpiani, ibid. 34, 10 (1982).

[2] J. Stat. Phys. 70, No. 1/2 (1993), special issue, stochastic resonance, edited by F. Moss, A. Bulsara, and M. F.
Schlesinger.

[3] M.I. Dykman, R. Mannella, P.V.E. McClintock, and N.G. Stocks, Sov. Phys. JETP Lett. 52, 141 (1990).

[4] N.G. Stocks, N.D. Stein, and P.V.E. McClintock, J. Phys. A 26, L385 (1993). 
[5] L.D. Landau and E.M. Lifshitz, Mechanics, 3rd ed. (Pergamon, Oxford, 1978).

[6] M.I. Dykman, R. Mannella, P.V.E. McClintock, and N.G. Stocks, Phys. Rev. Lett. 68, 2985 (1992).

[7] M.I. Dykman, R. Mannella, P.V.E. McClintock, N.D. Stein, and N.G. Stocks, Phys. Rev. E 47, 3996 (1993).

[8] L. Fronzoni, in Noise in Nonlinear Dynamical Systems, edited by F. Moss and P.V.E. McClintock (Cambridge University Press, Cambridge, England, 1989), Vol. 3, p. 222; and P.V.E. McClintock and F. Moss, in ibid. p. 243.

[9] The AD534 analog multiplier is from Analog Devices Inc., Box 9106, Norwood, MA 02062.

[10] B. McNamara, K. Wiesenfeld, and R. Roy, Phys. Rev. Lett. 60, 2626 (1988). 